\title{
The structure and function of intonational paragraphs in native and nonnative speaker instructional discourse
}

\author{
Lucy Pickering* \\ Department of English, 103 Morgan Hall, PO Box 870244, Tuscaloosa, AL 35487-0244, USA
}

\begin{abstract}
In the context of classroom communication, there is a premium on the clarity of the message, and instructors will typically employ multiple linguistic cues to highlight information structure. Using a model of intonation in discourse [Brazil, D. (1986). The communicative value of intonation in English. Birmingham, England: University of Birmingham, English Language Research. Brazil, D. (1997). The communicative value of intonation in English. Cambridge: Cambridge University Press.], this paper examines the use of intonational paragraphs as an organizational tool in the teaching discourse of native speaker teaching assistants and nonnative speaker international teaching assistants at a North American university. Analysis of the native speaker data presents evidence of intonational paragraphs defined by phonological criteria and used by the speakers to underscore local and global information structure. Comparative analysis of the parallel nonnative speaker data shows a considerably weaker control of intonational structure and a disturbance in prosodic composition that materially affects the comprehensibility of the discourse for native speaker hearers. The paper closes with a discussion of some of the implications of this study for international teaching assistant programs.
\end{abstract}

(C) 2003 The American University. Published by Elsevier Ltd. All rights reserved.

University faculties and graduate programs have become increasingly diverse. The numbers of international teaching assistants (ITAs) in scientific and technical fields such as engineering, mathematics, and laboratory sciences continue to grow, and the majority of US undergraduates are now likely to have important contact with international staff in their introductory courses. Despite the widespread creation of screening programs developed to assess the linguistic ability of these instructors,

* Tel.: + 1-205-344-4567; fax: + 1-205-348-1388.

E-mail address: lpickeri@bama.ua.edu (L. Pickering). 
communication failure between ITAs and their students is not uncommon, and the need for continued development of more effective educational programs remains acute. Researchers and practitioners who are focused on "the ITA problem" recognize the limitations of traditional pedagogical approaches and employ "integrated frameworks" that apply innovative work in discourse analysis to the particular issues presented by this group [see, for example, Madden \& Myers (1994) Discourse and Performance of International Teaching Assistants].

Using this approach, a range of linguistic information-structuring devices has been identified and investigated in studies of L1 and L2 academic discourse including rhetorical organization, lexical discourse markers, and syntactic complexity (Dudley-Evans, 1994; Rounds, 1987; Tyler, 1992; Tyler \& Davis, 1990; Tyler et al, 1988). An equally important linguistic strategy used by speakers to elucidate information structure in academic discourse is the creation of intonational paragraphs (Barr, 1990; Thompson, 2003).

The term "intonational paragraph" refers to a unit above the level of the tone unit and equivalent to the paragraph in written discourse (Lehiste, 1979). ${ }^{1}$ Due to differences in analytical approaches and to the investigation of a variety of discourse genres where these structural units may manifest themselves differently, speech paragraphs have been variously labeled as "major and minor paratones" (Brown, Currie, \& Kenworthy, 1980; Couper-Kuhlen, 1986; Yule, 1980), "pitch sequences" (Brazil, 1986, 1997), "sequence chains" (Barr, 1990), "phonological paragraphs" (Lehiste, 1979; Tench, 1996; Thompson, 2003), and "major tone groups" (Wichmann, 2000). A number of commonalities of both the structure and function of these units, however, unite these descriptions.

Analysts agree that speech paragraphs are produced and interpreted by speakerhearers using the phonetic cues that appear at their boundaries. The most prominent cues include a high pitch onset (as measured by fundamental frequency [Fo]) with an accelerated rate and volume, and a low pitch close possibly accompanied by laryngealisation, a drop in volume, and a narrowing of the pitch range (Brown et al., 1980; Lehiste, 1979; Wichmann, 2000; Yule, 1980). In addition to these boundary criteria, Tench (1996) suggests that there will be a "gradual descent" in pitch from the first to the final tone unit within a single speech paragraph, and Wichmann (2000) reports a similar tendency toward "supradeclination" within units in experimental data and broadcast news items. Changes in pitch level are accompanied by systematic variation in pause lengths. In perception studies of pause boundaries, Swerts and Geluykens (1993) found that longer pauses increased listener perception of boundary strength, and Cutler, Dahan, and Donselaar (1997) report studies in which speakers consistently produced pauses to create "paragraphs" in narrative retellings.

Both pitch resets and pause structure interact with other levels of discourse organization in the creation of speech paragraphs, most notably, topic structure. Production

\footnotetext{
1 By tone unit, I refer to the unit of intonational analysis traditionally recognized as consisting of a single intonation contour and containing a nuclear or tonic syllable. These are also referred to as sensegroups, breath-groups, tone-groups, intonation-groups, phonological phrases, or intonational phrases. For more detailed discussion, see Cruttenden (1997) and Wennerstrom (2001).
} 
studies in English and Dutch show that speakers use a higher pitch to initiate a new topic, a mid pitch level at points of topic continuation, and a low Fo at topic final boundaries or for asides or digressions (Nakajima \& Allen, 1993; Swerts \& Geluykens, 1994). Brown and Yule (1983) suggest that a speech paragraph is likely to begin with a topic expression which coincides with the high pitch onset, i.e. "an introductory expression to announce what (the speaker) specifically intends to talk about" (p. 101). Several analysts (Brown et al., 1980; Couper-Kuhlen, 1986; Wennerstrom, 2001) propose an even more complex hierarchical structure indicated by pitch level and range that correlates with the arrangement of sub-topics or "levels of embedding of a topic" (Wichmann, 2000: 130). Studies documenting the duration of pauses and topic structure suggest that the duration of pauses between utterances is longer for major than minor topic shifts (Brown et al, 1980; Lehiste, 1979; Swerts \& Geluykens, 1994).

This paper investigates the structure of intonational paragraphs and their relationship to topic development in the teaching discourse of North American teaching assistants (NS TAs) and Chinese international teaching assistants (ITAs). Analysis of the NS TA instructional monologues reveals a hierarchical series of phonologically defined units which coincide with structural boundaries at other levels of the discourse, thereby contributing to the overall organization of the teaching presentations. Comparative analysis of parallel ITA data demonstrates the inability of these speakers to effectively control this level of structural organization and examines how this may negatively impact the comprehensibility and effectiveness of ITA teaching presentations for a native speaker undergraduate population.

\section{Intonational paragraphs in $\mathrm{L} 1$ and $\mathrm{L} 2$ academic discourse}

Studies of L1 discourse that have investigated the role of prosodic structure in signaling global organization of a text suggest that speech paragraphs will be more prominent and perhaps more elaborate in lecture-style discourse (Swerts \& Geluykens, 1994; Tench, 1996; Thompson, 2003; Wennerstrom, 2001). Coulthard and Montgomery (1981) developed a ranked scale of structural units partially defined by phonological criteria to describe the semantic "chunks" of a typical lecture. Paragraph equivalent sequences are defined by an initial high pitch and a low closing pitch and comprise a series of clauses which are marked by some kind of "semantic coherence" (p. 34). Barr (1990) extends Coulthard and Montgomery's work in lecture monologue and has constructed a larger, phonologically-based unit termed a sequence chain which is defined both at its edges and internally by relative pitch height. She suggests that sequence chains parallel topic development, which she traces through the use of visual aids such as OHPs, handouts, and the blackboard. Both Coulthard and Montgomery and Barr base their analysis on Brazil's (1986, 1997) model of discourse intonation, which is also the theoretical framework used in the analysis reported here.

Comparative studies of L1 and L2 discourse structure focus on a variety of features of the intonation production of learners and demonstrate crucial differences in 
the use of prosodic cues by L2 speakers (Anderson-Hsieh, Johnson, \& Koehler, 1992; Pickering, 2001; Tyler et al., 1988; Tyler \& Davies, 1990; Wennerstrom, 1997). In a study investigating 30 intermediate learners from three L1 backgrounds performing a read-aloud task, Wennerstrom (1994) found that while Spanish L2 speakers and a native speaker control group marked a new topic with a high pitch onset, Thai and Japanese L2 learners demonstrated no paragraph initial pitch change. In a follow-up 1998 study, Wennerstrom examined 10-12 min lectures given by 18 speakers of Mandarin Chinese and found a significant correlation between the students' scores on a spoken exam and their ability to indicate rhetorical units by expanding their pitch range (Wennerstrom, 1998).

In addition to pitch variation, studies investigating fluency (i.e. pause structure and hesitation phenomena) in L2 discourse show qualitative differences in both placement and length of pauses as compared to an L1 model. Riggenbach (1991) and Anderson-Hsieh and Venkatagiri (1995) found more nonlexical fillers and unfilled pauses in non-fluent NNS speech, and long pauses frequently appeared within rather than between intonation units. In ITA lecture extracts, Rounds (1987) found that pauses were both longer and more erratic than those in the NS data and tended to regularly break up conceptual units. As Griffiths (1991) notes, studies in pausology, particularly as they relate to L2 discourse, can be difficult to reconcile. Differences in methodology and contrasting speech genres make it difficult to generalize results and predict lengths and placement. Rounds does not consistently specify values in her discussion of pauses, and Brazil does not elaborate on pause patterns apart from noting that they may and frequently do coincide with tone unit boundaries. For the purposes of this study, I have used a model identifying pause defined units developed by one group of researchers (Brown, 1977; Brown, Currie, \& Kenworthy, 1980; Brown \& Yule, 1983). They identify three major groups: "topic pauses" of $0.8 \mathrm{~s}$ and longer which "clearly coincide with major semantic breaks" (p. 56); "substantial pauses" of between 0.6 and $0.8 \mathrm{~s}$ which tend to coincide with single contours; and "very short" pauses which vary between 0.2 and $0.4 \mathrm{~s}$ and frequently co-occur with incomplete syntactic structures. Pauses of less than $0.2 \mathrm{~s}$ are considered as "articulatory pauses" following Griffiths (1991). As these researchers were working with spontaneous speech rather than lecture discourse, this model was taken as a first approximation for pause analysis in the data. Using an integrated framework of discourse intonation and content structure, this study examines both pitch and pause structure in parallel L1 and L2 teaching discourse.

\section{Theoretical framework}

Brazil's model of intonation in discourse comprises three interacting systemstone, key, and termination - which are manifested on the prominent syllables in the tone unit, i.e. those syllables that are distinguished from the surrounding content by fundamental frequency ( $\mathrm{Fo}$ ) excursions. The tone system is familiar from traditional descriptions of English intonation (Crystal, 1969; Halliday, 1967; Tench, 1996) and describes the pitch movement (typically either a rise or fall) that is carried on the 
nuclear syllable. The key and termination systems describe the pitch level of the prominent syllables and are crucial in the identification of speech paragraphs as defined by pitch height. Key choice is realized on the onset syllable (the first prominent syllable in the tone unit) and termination choice on the nuclear syllable. In cases where there is only one prominent syllable in the tone unit, both key and termination choice fall on the same syllable, as shown in the examples below.

Both key and termination choices are analyzed under a three term system that divides the speaker's pitch range into three levels: high, mid, and low. ${ }^{2}$ Following the first onset key, subsequent pitch levels on prominent syllables are identified as appreciably "higher than" or "lower than" the preceding key or termination choice. Each choice is glossed with a particular communicative value. High key denotes the constituent (or matter of the tone unit) as either "contrastive", with something derivable from the preceding discourse, or "particularized", i.e. highlighted as crucial over and above the surrounding information (see Appendix A for transcription conventions):

//he took the exAM // and $\uparrow \underline{\text { FAILED } / / ~}$

He did not pass, as you might have expected: contrastive. (Sinclair \& Brazil, 1982: 144)

In a conversational exchange, a high termination has an adjudicating function which constrains the second speaker to a matching 'Yes/No' response. Mid-level key choices have an additive function and denote the constituent as an "expansion" of the information in previous units:

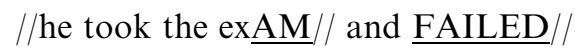

He did both: additive. (Sinclair \& Brazil, 1982: 144)

A mid-level termination choice carries the speaker's expectation that the hearer will concur with the utterance. Finally, a low pitch choice signifies an "equative" value in relation to previous units, i.e. an indication that no new information is added:

//he took the exAM $/ /$ and $\downarrow$ FAILED $/ /$

As you would expect; from what you know of him you will assume that taking it involves failing it: equative. (Sinclair \& Brazil, 1982: 144)

Low termination choices carry an additional restrictive function as they cue the completion of a unit.

\footnotetext{
2 Clearly pitch level is a gradient feature. For any given speaker, an indefinite number of absolute pitch levels may be identified. Pitch level may also be affected by a number of factors including individual idiosyncracies, emotional involvement (Bolinger, 1988) or sociocultural convention (van Bezooijen, 1995). However, once we abstract away from these factors, Brazil proposes that we are left with a small number of pitch levels used to carry linguistic contrasts.
} 
It is the inclusion of key choice, or relative onset level, that provides a principled framework for the description and interpretation of prosodic units larger than the tone unit. Brazil proposes a paragraph unit termed a pitch sequence. This is a stretch of consecutive tone units that fall between two low termination choices; they typically delimit longer sections of speech and comprise a semantically coherent group of tone units. In a monologue, a speaker may divide the discourse into a series of pitch sequences by following each low termination with a high, mid or low key choice and thereby opening a new unit. Pitch sequence openings carry similar communicative value as key choices within tone units. A low termination pitch sequence closure followed by a high key pitch sequence opening marks a point of maximal disjunction from the previous sequence and normally coincides with major semantic or structural boundaries in the discourse. A mid key pitch sequence carries a value of enlargement or addition to the preceding sequence, and a low key sequence is associated with reformulations or asides which typically have a reduced pitch range (Beckman, 1997; Tench, 1996). Fig. 1 illustrates a high key pitch sequence followed by a mid key pitch sequence, which is added after a low key aside.

In this chemistry lab, the NS TA is demonstrating the procedures the students will use to conduct a Thin Layer Chromatography (TLC) experiment. The first pitch sequence begins with the high key focusing phrase "now the first thing you wanna do" as the TA begins describing how to mark the TLC plate. Following the tone units "there's rulers in the stockroom," there is a low terminating aside - "and I don't have a pencil with me" (line 3)-as the TA realizes that he does not have a pencil to demonstrate exactly what the students should do. This low termination closes the first pitch sequence and is followed by a new mid key sequence opening: "but what you wanna do is." The mid key choice matches the topic continuation (the first thing to do with your plate is to mark it) that was cut short by the aside.

In recent work that applies Brazil's model to the analysis of native speaker lecture discourse, Barr (1990) formalizes a group of pitch sequences into a larger unit of intonation structure she terms a sequence chain:

[The sequence chain] is above the pitch sequence and is defined as a string of pitch sequences such that the first pitch sequence and only the first sequence begins with a high key or a lecturing frame. Thus minimally, a sequence chain

\begin{tabular}{|c|c|}
\hline & $/ / /$ now the $\uparrow$ FIRST thing you wanna $\underline{\mathrm{DO} / /(0.5) / / \text { WITH your tlc } \underline{\mathrm{PLATE}} / / \text { is }}$ \\
\hline & $\uparrow$ HOPEfully you've got a PENcil// alRIGHT and they'll there's $\uparrow \underline{\text { RULers } / /(0.7)}$ \\
\hline & //in the STOCKroom and i don't have a PENcil with $\downarrow \underline{\mathrm{ME}} / / /(0.75) / / /$ but what \\
\hline & you wanna DO is you wanna $\uparrow \underline{M A R K} / /$ at least a $\uparrow$ ONE centimeter LINE// (0.2) \\
\hline & //down the BOTtom of this PLATE// \\
\hline
\end{tabular}

Fig. 1. Pitch sequence structure from an NS TA chemistry presentation. 
consists of a single high key-initial pitch sequence, but maximally, a sequence chain consists of indeterminate numbers of pitch sequences such that any noninitial pitch sequence is either mid/low key and therefore additive or equative to the previous one. (p. 11)

Thus at points of maximal disjunction following a low termination choice, Barr proposes that the speaker may initiate a new phonological paragraph comprising one or more pitch sequences through opening in a high key or with a high or mid key lecturing frame such as //SO//, //NOW//, //RIGHT//, or //OK//. This hierarchical relationship of tone unit, pitch sequence, and finally sequence chain is corroborated by Couper-Kuhlen (1986), who suggests that "minor paratones" which open in a high key may initiate "major paratones" (p. 193).

In the same way in which Brazil proposes that pitch sequence boundaries match a semantically coherent group of units, Barr suggests that topic-based criteria will match sequence chain phonological cues. In data from four college-level lectures, she found that sequence chain boundaries were marked with an introductory topic expression and were coextensive with the level of lecture organization found in the layout of prepared visuals such as overhead projections, the blackboard or handouts. Sequence chain boundaries also paralleled changes in "discourse plane" (Sinclair \& Brazil, 1982); that is, shifts in the area of attention of the discourse such as a movement from talk about the content of the class to talk about the organization of the class.

Due to the particular style of classroom discourse examined in the present study, there was some difficulty in applying Barr's criteria of co-occurring visual cues as support for coinciding phonological and semantic structural boundaries at the level of sequence chain structure. Barr focused on concept-based lectures in which professors used a variety of prepared visual aids. The data I investigate are typical of the style of short prelab presentations given in introductory science laboratory classes (Jacobson, 1986). They are less formal in nature, and there are no accompanying prepared materials. TAs used only the blackboard as a visual aid, and usually in a less systematic manner than might be found in a longer, more formal lecture.

In order to map co-occurring structural boundaries at other levels of the discourse organization, in place of Barr's original criteria of prepared visuals, I have drawn on the transaction structure proposed by Coulthard and Montgomery (1981) and Shaw (1994). A transaction is a "chunk" of discourse containing a unified topic and defined by prospective and retrospective focusing markers at its boundaries. In an analysis of NS teaching discourse in university classes, Shaw suggests that typical prospective and retrospective markers include both verbal and non-verbal cues, e.g. lexical phrases such as "for the first part", boundary micro-markers such as "ok," (equivalent to Barr's lecturing frames), and topic length pauses. Shaw's analysis of possible phonological boundary cues, however, is limited to a brief discussion of the use of rising or falling intonation on evaluation markers. In this study, I have united both Barr's and Shaw's findings in order to examine the co-occurrence of phonological cues indicating sequence chain structure with lexical and nonverbal transaction boundary cues. Places where these are coextensive are seen to be evidence of the 
speaker's intention to signal a maximal structural boundary. An example of coextensive transaction and sequence chain boundaries is shown in Fig. 2.

This extract, from the same NS TA presentation given in Fig. 1, shows a series of two adjacent sequence chains and the opening marker of a third. The boundary markers separate a series of instructions given to the students concerning the equipment they will be using for the experiment. The first sequence chain is also marked as a transaction boundary with two prospective focusing markers in a high key: "Ok for TLC you're gonna need several pieces of equipment" and "first off." The closing boundary for the transaction and first sequence chain co-occur with the recapitulation statement, "you're gonna make your own little developing chambers" (lines 5-6), which ends in a low termination and is accompanied by a topic length pause. In the second sequence chain, the TA moves from discussing the developing chamber to the chemical solvent and marks this with a high key prospective marker: "ok the solvent you're gonna use." The sequence chain ends with a low key recapitulation, "so it's there if you forget what solvent system to use" (lines 8-9), cooccurring with a low termination and a topic length pause. The final concurrent sequence chain and transaction boundary occurs with the prospective lecture frame "ok." An initial model summarizing the interaction between intonational structure and co-occurring structural boundaries in the NS TA teaching discourse is shown in Appendix B.

\section{Method}

In large state universities in the USA, large undergraduate science lecture classes are typically broken up into smaller sections for lab work or problem solving work.

\begin{tabular}{|c|c|}
\hline 1 & ////ok for $\uparrow \mathrm{Tl} \underline{\mathrm{C}}$ you're gonna need $\uparrow \underline{\mathrm{SEv}}$ eral // $(0.68) / /$ pieces of eQUIPment// \\
\hline 2 & 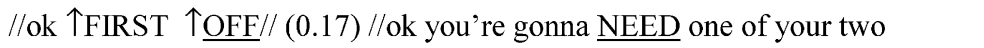 \\
\hline 3 & hundred and FIFty milliliter BEAkers// $(0.57)$ and one of your WATCH \\
\hline 4 & glasses// (0.5) //ok this is gonna be now your developing CHAMber for the// \\
\hline 5 & $(0.45) / / \downarrow \underline{\mathrm{UM}} / /(0.74) / / \mathrm{Tl} \underline{\mathrm{C}} / /(0.57) / /$ you're gonna make your OWN little \\
\hline 6 & developing $\downarrow$ CHAMbers//// (1.14) ////ok the $\uparrow$ SOLvent you're gonna USE \\
\hline 7 & to deVElop the $\uparrow \underline{P L A T E S} / /$ is $\uparrow$ ethoLAcetate// and this $\uparrow$ IS in the $\uparrow$ NOTES $/ /$ \\
\hline 8 & $(0.4) / / \underline{\mathrm{SO}} / /(0.82) / /$ it's THERE if you forget what SOLvent system to \\
\hline 9 & $\downarrow \underline{\mathrm{USE}} / / / /(0.82) / / / / \mathrm{o} \underline{\mathrm{K}} / / \ldots$ \\
\hline
\end{tabular}

Fig. 2. Coextensive sequence chain and transaction boundaries from an NS TA presentation. 
Each section covers the same material but is taught by a different TA. Prior to the students beginning their experiments, the TA will usually give a short presentation. Typical functions performed by TAs in these prelab presentations and discussion sections include giving theoretical background, reviewing homework, explaining relevant terms or equations, and demonstrating experimental procedures (Axelson \& Madden, 1994; Jacobson, 1986). For this study, 12 2-4 min extracts from naturally occurring classroom presentations in introductory lab sections and math discussion sections taught by six male North American NS TAs and six male Chinese ITAs were analyzed. To ensure that the extracts involved equivalent presentations of the same material, I recorded the opening 2-4 min of each presentation by the NS TAs and ITAs from the same subject area on the same day or in the same week. The six ITAs were from mainland China, and their L1 was Mandarin Chinese. Each had received a score of 45-50 on the ETS Speaking Proficiency English Assessment Kit (SPEAK). This indicates their communication skills to be "somewhat to generally effective" (Educational Testing Service, 1996, p. 9). The six NS TAs were described as "relatively experienced," but none was specifically described as a "model" TA. The data set is summarized in Table 1.

The data were recorded in the classroom on audio- and videotape using a Sony TCD-D8 Digital Audio Tape-corder (DAT), a Sharp VL-L49OU VHS Camcorder, a Telex FMR-150c Wireless system, and a Telex SCHF745 headset microphone. The DAT recordings were transferred to a Kay Elemetrics Computerized Speech Laboratory (CSL) Model 4300, and fundamental frequency traces were computed for all the data using the pitch extraction function of the CSL. Intonation choices were identified through both auditory and instrumental analysis (SchuetzeCoburn, Shapley, \& Weber, 1991; Watt, 1997). The key and termination choices were identified and interpreted within the systems given in Brazil's (1986, 1997) model and transcribed using those conventions. The analysis considers sequence chain and pitch sequence structuring in the group of NS TA presentations followed by a comparative analysis of these units as they appear in the parallel ITA presentations.

Table 1

Summary of teaching presentations

\begin{tabular}{llll}
\hline Subject & Topic & Participants NS TAs & ITAs \\
\hline Chemistry & Scheme for unknown analysis & TAC-1 & ITAC-1 \\
& ITAC-2 \\
& Thin layer chromatography & TAC-2 & ITAC-3 \\
Math & Exponential growth and decay & TAM-1 & ITAM-1 \\
& & & ITAM-2 \\
Physics & Torques and forces in equilibrium & TAP-1 & ITAP-1 \\
Electrical Engineering & Drawing a Bode plot & TAP-2 & TAE-1 \\
\hline
\end{tabular}

a There is no parallel extract for TAE-1, as no Chinese ITAs were teaching in this section at the time of recording. 


\section{Results}

\subsection{NS TA discourse}

\subsubsection{Sequence chain structure}

In the six presentations examined here, 34 complete sequence chains were identified by the intonational criteria of relative pitch level. Typically, sequence chains comprised an opening boundary in one tone unit followed by a number of tone units containing a topic expression and development, and a final tone unit or small group of units forming a closing boundary. Twenty-four of these were also marked as transaction boundaries with both prospective and retrospective marking by non-prosodic means, and a further six sequence chains co-occurred with either prospective or retrospective markers. A summary of co-occurring transaction boundaries is shown in Table 2.

The remaining four sequence chains coincided with shifts in discourse plane, i.e. moments where the teacher moved from "telling something" to "talking about telling something" or to "asking something."

Examples of co-occurring transaction and sequence chain cues were shown in Fig. 2 from TAC-2's presentation. Fig. 3 illustrates a sequence chain boundary coextensive with a shift in discourse plane in a series of two adjacent sequence chains from TAP-2's presentation. The TA begins the physics laboratory class with a discussion of the prelab that the students completed as homework. The first sequence chain opens with a high key focusing expression, "so you guys had problems with the prelab right," followed by the TA finding the relevant page in the textbook and reading aloud the question, which closes with a low termination and extended pause (line 5). During the $4.85 \mathrm{~s}$ pause there is a shift in discourse plane as TAP-2 moves to the blackboard and opens a new sequence chain with a high key lexical phrase, "the way this thing goes is" (lines 5-6), as he begins to explain the question. These cooccurring lexical, prosodic, and topic-related boundary cues are further strengthened by the behavior of the TA, who clearly scans the audience before moving toward the blackboard and beginning a new sequence chain.

Table 2

Transaction boundaries in the NS TA presentations

\begin{tabular}{lc}
\hline Transaction boundary markers & Number of Occurrences \\
\hline Prospective markers & 28 \\
High key lexical phrases & 10 \\
Mid/High key micro-markers & 15 \\
High key topic statement & 3 \\
Retrospective markers & 29 \\
Low termination recapitulation & 7 \\
Statements & \\
Low termination micro-markers & 11 \\
Topic length filled/unfilled pauses accompanied by low termination & 11 \\
\hline
\end{tabular}




\begin{tabular}{|c|c|}
\hline 1 & 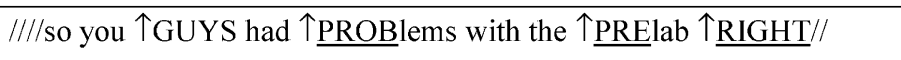 \\
\hline 2 & [1.9] //AND the FIRST question WAS uh// [4.42] //QUEStion number \\
\hline 3 & 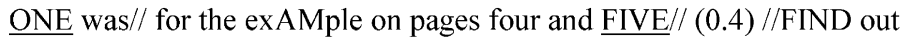 \\
\hline 4 & 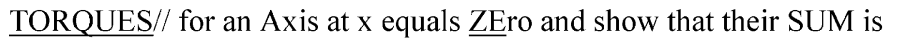 \\
\hline 5 & still $\underline{\mathrm{ZEro} / /}(1.95) / / \downarrow \underline{\mathrm{UH}} / /(4.85) / / / / / / /$ the $\uparrow W A Y$ this// (0.7) //thing \\
\hline 6 & GOES... \\
\hline
\end{tabular}

Fig. 3. Coextensive sequence chain and plane change boundary in TAP-2's presentation.

In addition to establishing an interaction between prosodic and topic based units of organization, these data also minimally substantiated Barr's original criteria of matching visual cues to sequence chain structure. Table 3 shows real-time board work coinciding with sequence chain boundaries in TAC-1's teaching discourse.

The TA is reviewing the procedures that students need to complete in order to conduct an analysis of an unknown chemical compound. Each topic expression opens a new sequence chain and is matched by its visual representation on the blackboard.

Throughout the NS TA presentations, the cluster of cues provided by the cooccurring transaction, plane change, and sequence chain boundaries underscore points at which the structural and semantic links binding one group of pitch sequences or tone units are completed. Internally, the sequence chains may also incorporate a series of smaller units that emphasize connections between individual utterances. The following section examines the pitch sequence structure created by the NS TAs within sequence chains and how this interacts with topic structure.

\subsubsection{Pitch sequence structure}

The reader will recall that pitch sequences are the interposing unit of prosodic structure between the sequence chain and the tone unit. They differ from sequence

Table 3

Visual cues coinciding with sequence chain structure in TAC-1's presentation

\begin{tabular}{ll}
\hline Opening tone units of three sequence chains (TAC-1) & Boardwork \\
\hline //// But FOR our unKNOWN we HAVE & $\mathrm{Na}+, \mathrm{K}+, \mathrm{Nh} 4+, \mathrm{OH}-, \mathrm{NO} 3-, \mathrm{Cl}-, \mathrm{HsO} 4-$ \\
SEven ions we have to TEST for// & \\
$\begin{array}{l}\text { ////One of the FIRST things that we did } \\
\text { was a FLAME test } / /\end{array}$ & 1. Flame Test \\
$/ / / /$ the SEcond set of TESTS we did was// & 2. Colbaltinitrate Test \\
$/ /$ that cobaltiNItrate $\underline{\text { TEST } / /}$ &
\end{tabular}


chains in that they may begin with a high, mid, or low key choice. In the NS TA presentations, the number of pitch sequences per sequence chain varied widely both within and between speakers. Internally, pitch sequences also ranged in length from one tone unit containing a low termination marker to much longer sequences integrating a number of tone units. Qualitative analysis of the kinds of information typically contained within pitch sequences suggested that the units were intentionally created by the NS TAs to emphasize structural boundaries between different kinds of classroom content. Most commonly, pitch sequences distinguished between two overall types of content: main and subsidiary (Coulthard \& Montgomery, 1981). Main discourse describes the informative content of the presentation, while the category of subsidiary content subsumes a variety of types of teaching language, from short glosses or asides to much longer segments of discourse concerned with the organization of the class. An example of this latter type of subsidiary content and its interaction with pitch sequence structure, taken from TAC-1's chemistry presentation, is shown in Fig. 4.

The extract shows the opening sequence chain of the presentation, which is made up entirely of subsidiary content, followed by the beginning of the second sequence chain marking the shift to the main discourse or informative content, "for our unknown we have seven ions we have to test for" (line 11). Within the first sequence chain, the content of the first pitch sequence can be glossed as "getting the students' attention" by first framing and focusing a topic expression, "OK, begin about

\begin{tabular}{|c|c|}
\hline 1 & 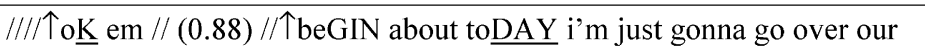 \\
\hline 2 & unknown aNAlysis $\underline{\mathrm{SCHEME}} / /(0.14) / / \cos$ it'll BEnefit anyone who's \\
\hline 3 & going to need to be WORKing on it// (0.07)//so if you wanna gather \\
\hline 4 & $\underline{\text { ROUND }}$ i'm gonna do it up here on the $\downarrow_{\text {BOARD }} / / /(1.17) / / /$ if you \\
\hline 5 & HAVE $\underline{\text { ONE }}$ it's a great time to check to see if you $\downarrow_{\underline{L I K E}}$ it/// (2.42) \\
\hline 6 & ///uh before i $\underline{\text { START }} \uparrow E V$ erybody has MADE it up to at LEAST section \\
\hline 7 & FOUR on lab $\uparrow \underline{T W O}$ everybody's made it at LEAST that $\underline{\text { FAR }} \underline{\text { RIGHTT// }}$ \\
\hline 8 & $(0.36) / / \mathrm{exCEPT}$ for $\downarrow \underline{\mathrm{YOU}} / / /(0.88) / / / \mathrm{GOOD} / /(0.07) / /$ that's about as \\
\hline 9 & FAR as it's NEcessary// (0.5) //cos i'm BAsically only gonna go over our \\
\hline 10 & POsitive IONS $(0.73) / /$ and BRIEFly over the $\downarrow$ NEgative ions//// (1.0) \\
\hline 11 & ////but $\uparrow F O R$ our $\uparrow$ unKNOWN... \\
\hline
\end{tabular}

Fig. 4. Interaction between main and subsidiary content and pitch sequence structure from TAC-1's presentation. 
today," followed by a mid key invitation to the students to gather round the board (line 4). Both the second and the third pitch sequences add further subsidiary content in the form of a comment ("if you have one it's a great time to see if you like it" [line 5]) and a procedural aside to check that the students are ready to begin the chemical analysis (lines 7-8). A high adjudicating key is used for the conducive question, "everybody has made it up to at least section four on lab two?" (lines 6-7), followed by a mid key repetition. This pitch sequence ends with the low key aside, "except for you" (line 8). The TA's use of equative low key reflects the parenthetical nature of the comment, which is addressed to one student in particular rather than the whole group. Following the aside, TAC-1 raises the key of his next tone unit, "good" (line 8), to open a new mid key pitch sequence directed back toward the group as a whole. The last pitch sequence adds a final comment regarding what TAC-1 will cover in his presentation: "cos I'm basically only gonna go over our positive ions and briefly over the negative ions" (lines 9-10). Immediately following this pitch sequence closure, which simultaneously closes the sequence chain, the TA opens a new sequence chain marking a shift in discourse plane from a focus on class organization to the chemistry content.

This extract is a somewhat complex example of the possible interaction between subsidiary content and pitch sequence structure. More frequently, pitch sequence boundaries separated one piece of subsidiary content from the main content surrounding it. This often happened when the NS TAs were temporarily distracted by board work or when they were demonstrating equipment. In Fig. 1, for example, the low terminating aside, "I don't have a pencil with me," closes the original pitch sequence, and TAC-2 opens a new pitch sequence in a mid key to complete the informative content that was briefly interrupted.

\subsection{IT A discourse}

\subsubsection{Sequence chain structure}

Although it was possible to identify sequence chains in the ITA data, these phonologically defined paragraphs did not consistently create a coherent level of structural organization, and it would be misleading to suggest that they fulfilled the same function as they did in the NS TA teaching data. Boundaries marked by phonological cues were frequently not matched by a cluster of cues at co-occurring levels of the discourse structure, and disruption in the internal structure of the units often rendered them less effective. Thus, despite the appearance of a sequence chain structure in the ITA presentations, phonological cues in many cases did not actually mark maximal points of disjunction and could not be reliably used by the NS hearer as an organizational cue.

Analysis of prosodic units defined primarily by pitch level was hampered at the outset by a compression of overall pitch range in the ITA teaching presentations as compared to the pitch ranges found in the NS TA data set. In the NS group, although individual movements between the three levels of key varied both within and between speakers, the overall pitch range of all six speakers was approximately 


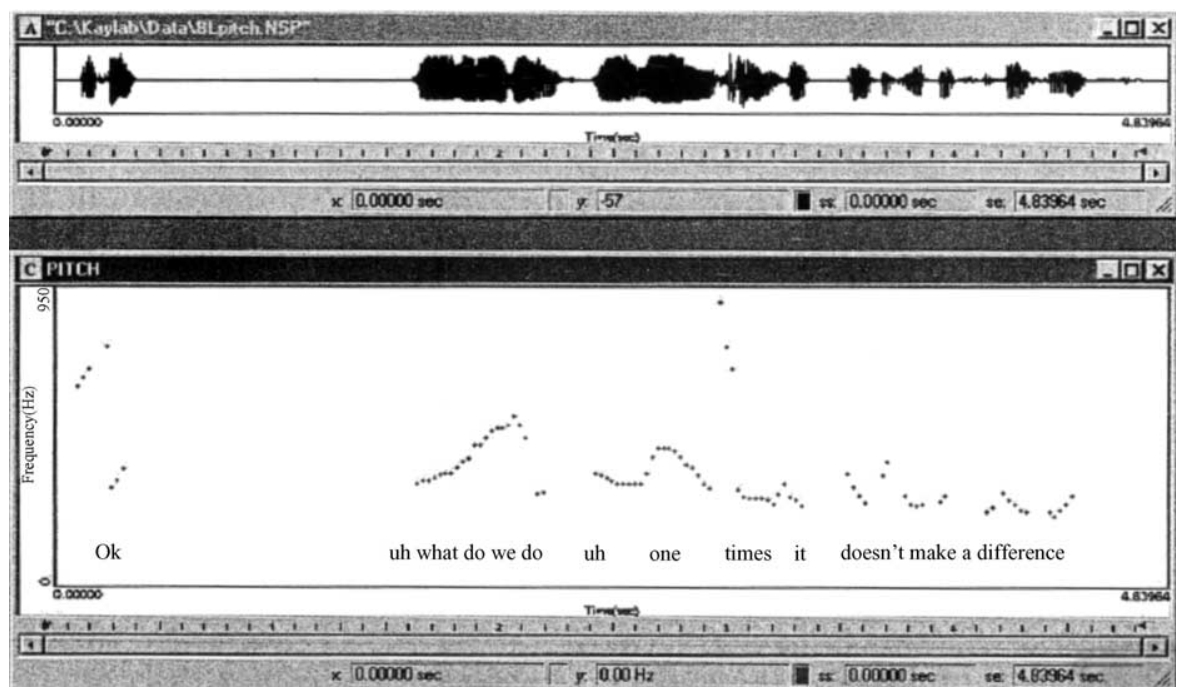

Fig. 5. Fo reading of a sequence chain boundary from TAM-1 math presentation.

$50-250 \mathrm{hz}$, with a median frequency of 100-150 hz. ${ }^{3}$ Sequence chains often began with a heightened pitch value or at a level higher than any value internal to the sequence chain, which contributed to the perception of a maximal boundary. In contrast, in the ITA data, overall pitch ranges were more variable and tended to be smaller (approximately 100-200 hz). As a result, pitch intervals between prominent syllables were less distinct, and pitch peaks were often less pronounced. Comparative examples of Fo readings at the opening of a sequence chain from a NS TA math presentation and parallel ITA presentation are shown in Figs. 5 and 6. Fig. 5, taken from TAM-1's presentation, demonstrates a heightened pitch level at the opening of the unit, "what we DO," followed by a steady decrease in the heights of the pitch peaks. Fig. 6 shows a maximal boundary from the equivalent ITA presentation given by ITAM-1. A transaction boundary is suggested by a retrospective marker just prior to these units, "that is the definition of half life," which closes with a low termination followed by a $1.68 \mathrm{~s}$ pause. The opening marker of a new unit, "so we have," is minimally projected by pitch height, as the Fo reading shows.

Despite these reduced ranges, a minimally fixed framework, i.e. a typical pitch range for a given speaker (Couper-Kuhlen, 1986), was identified for each ITA so that it was possible to distinguish higher key choices, which were then used to determine sequence chain structure. Thirty-seven sequence chains were identified in the ITA data, and a summary of co-occurring transaction boundary markers is shown in Table 4. Prospective and retrospective markers were similar in lexical content to those used by the NS TA group; however, there were fewer markers overall: only $51 \%$ of initial boundaries and $62 \%$ of final boundaries are marked in the ITA presentations compared to $82 \%$ of initial boundaries and $85 \%$ of closing

\footnotetext{
${ }^{3}$ Crystal (1987) notes that the typical fundamental frequency of an adult male is around $120 \mathrm{hz}$.
} 


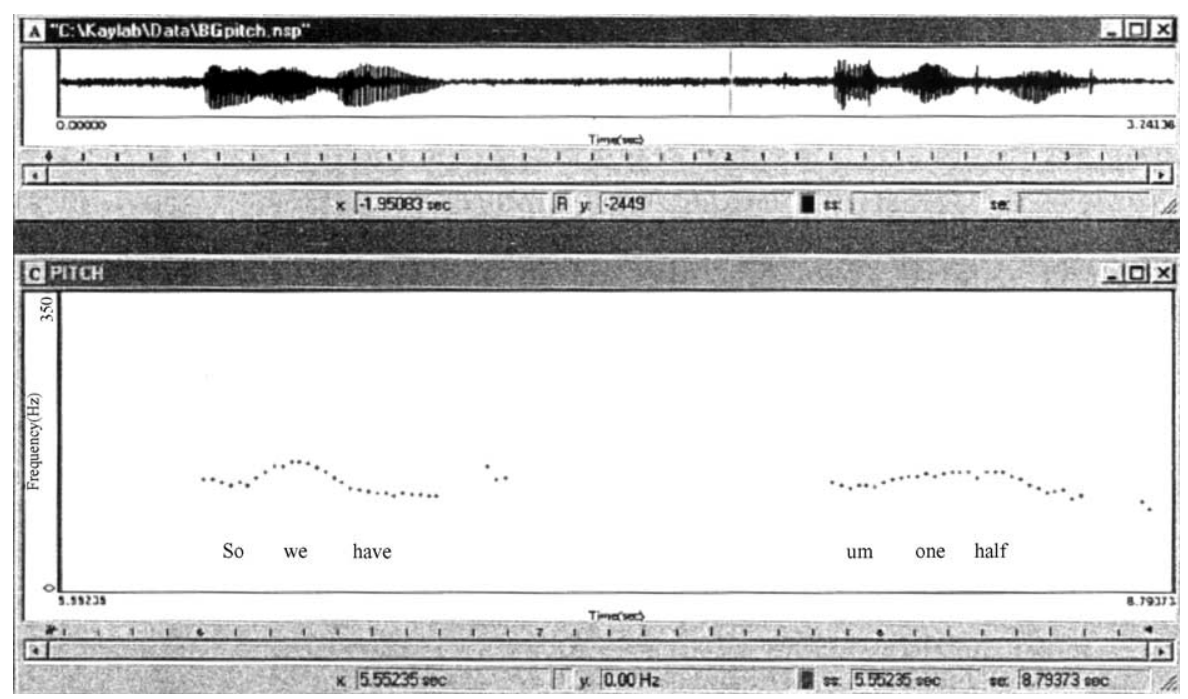

Fig. 6. Fo reading of a sequence of chain boundary from ITAM-1 math presentation.

Table 4

Transaction boundaries in the ITA data

\begin{tabular}{lr}
\hline Transaction boundary markers & Number of occurences \\
\hline Prospective markers & 19 \\
High key lexical phrases & 9 \\
Mid key micro-markers & 10 \\
& 23 \\
Retrospective Markers & 7 \\
Low termination recapitulation & \multicolumn{2}{|c}{} \\
Statements & 9 \\
Low termination micro-markers & 7 \\
Topic length filled/unfilled pauses accompanied by low termination & 7 \\
\hline
\end{tabular}

boundaries in the NS TA group. In addition, focusing markers were less prosodically distinct in the ITA data, and long internal silences fractured larger organizational units and resulted in difficulties in assessing retrospective pause boundaries. In the NS TA data set, topic length pauses $(0.8-1+\mathrm{s})$ were reliable indicators of a boundary or were clearly connected to pauses while the NS TA engaged in board work or equipment demonstrations (see, for example, Fig. 3). In contrast, the ITA presentations were characterized by frequent, random silences of varying lengths which were classified as "empty silences" (Rounds, 1987: 652). Fig. 7 shows the last unit of one sequence chain $(/ / \downarrow \mathrm{SO} / / / /)$, and the next complete sequence chain from ITAC-1's presentation. The complete sequence chain contains a number of paragraph-internal topic length pauses, (1.67), (0.95), (1.0), (0.99), (1.14), (0.95), before closing with a low key and pause boundary markers of a similar length, (1.7) and (1.2). 


\begin{tabular}{|c|c|}
\hline 1 & $(0.85) / / \downarrow \underline{\mathrm{SO}} / / / /(1.17) / / /$ this $\uparrow \mathrm{TWO}$ step is very $\underline{\mathrm{EAsy}} / /(0.4) / / \uparrow \mathrm{THEN}$ \\
\hline 2 & 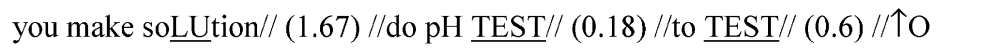 \\
\hline 3 & 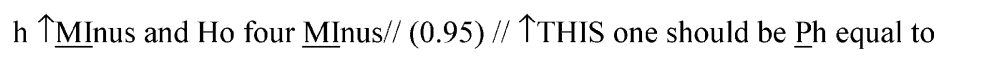 \\
\hline 4 & THREE// (1.0) // if $\uparrow$ THIS// (0.14) //if your $\uparrow \mathrm{Ph}$ is $\uparrow$ THREE// $(0.26) / / \mathrm{er}$ \\
\hline 5 & 个thirTEEN// (0.99) //that means THIS one is NOT there// (0.48)//because \\
\hline 6 & $\uparrow \mathrm{THIS}$ one $\uparrow$ canNOT// $(0.33) / /$ present at the $\uparrow \mathrm{SAME} \underline{\mathrm{TIME} / /(0.66) / / \text { if }}$ \\
\hline 7 & your $\mathrm{Ph}$ is// (0.26) //aROUND $\uparrow \underline{\text { TWO }}$ that means $\uparrow$ THIS// (0.48) //个THIS \\
\hline 8 & should be THERE// (1.14)//that $\uparrow$ ALso means $\uparrow$ THIS is NOT there// (0.95) \\
\hline 9 & because THIS// (0.26) //these two cannot present at the SAME $\downarrow \underline{T I M E} / /(1.7)$ \\
\hline 10 & $/ / \downarrow$ AND er//// (1.2) \\
\hline
\end{tabular}

Fig. 7. Problematic pause structure and sequence chain boundary markers from ITAC-1's presentation.

In those places where the ITA group approximated the NS model in their use of boundary marking, transaction structures were frequently not comparable in terms of internal structure. In the NS TA data, sequence chains typically consisted of focusing activity at the opening boundary, followed by a topic expression and its development, and a closing boundary. No similar consistent pattern was found in the ITA data. In many cases, the topic expression did not match the material in the sequence chain, or the sequence chain consisted of only a partial topic expression and no development; in some cases the topic statements themselves were unintelligible. Fig. 8 shows an extract from ITAC-1's presentation that directly corresponds to the extract from TAC-1's presentation shown in Fig. 3.

The first sequence chain opens the presentation and begins by getting the students' attention. It is well-formed with both a prospective marker, "ok students," and a recapitulation statement, "just wait a minute then continue your lab" (line 5). The second sequence chain consists of only the topic statement, "today we make two sample for your unknown test" (lines 6-7), and as such appears to indicate a plane change between the administrative organization of the lab and the actual chemistry content; in fact, in the third sequence chain the ITA returns to the organization of the presentation: "this is my suggestion for this unknown test" (line 9). Sequence chain four begins with a repeat of the earlier topic statement, "first you will have two samples" (lines 10-11); however, this is again followed by organizational instructions: "because this is a time limit so I suggest you make sure how to do this first." Finally, the same topic expression is repeated for the third time, "you get two sample it's a solid" (lines 15-16), and accurately marks a plane change to the 


\begin{tabular}{|c|c|}
\hline 1 & 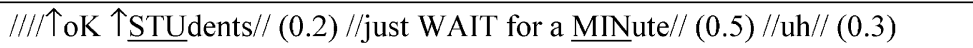 \\
\hline 2 & 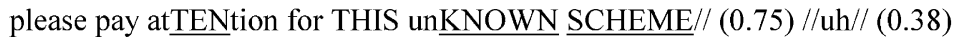 \\
\hline 3 & $/ / \uparrow \mathrm{eSPECial}$ for $\underline{\mathrm{YOU}} / /(0.09) / / \mathrm{DO}$ this un $\underline{\mathrm{KNOWN}}$ uh toDAY// $(0.57)$ it's \\
\hline 4 & $\uparrow A L s o$ good for you to do NEXT WEEK// (0.09)//if you// $(0.2) / / \underline{\mathrm{SO}} / /(0.38)$ \\
\hline 5 & just wait a $\underline{\mathrm{MIN} u t e}$ then conTINue your// $(0.75) / / \mathrm{er} \downarrow \underline{\mathrm{LAB}} / / / /(0.38) / / \mathrm{er}$ \\
\hline 6 & $\uparrow$ toDAY we $\uparrow$ MAKE have $\uparrow$ TWO SAMple// (0.09) //for your $\downarrow$ unKNOWN \\
\hline 7 & $\downarrow \underline{\text { TEST } / / / /(0.94) / / / / \uparrow T H I S ~ a-~ a l l ~ S T U F F ~ i s ~ a-/ / ~(0.09) / / w e ~ h a v e ~ L E A R N E D ~}$ \\
\hline 8 & 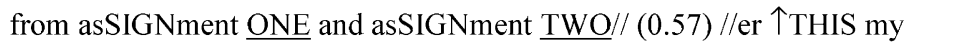 \\
\hline 9 & 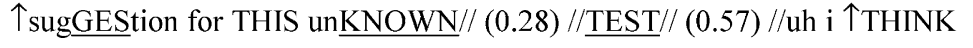 \\
\hline 10 & itss it will SAVE you $\downarrow \underline{\text { TIME}} / / / /(0.94) / / / /$ uh $\uparrow$ FIRST you $\uparrow \underline{\text { WILL }}$ er have \\
\hline 11 & 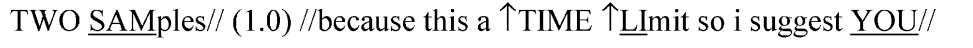 \\
\hline 12 & 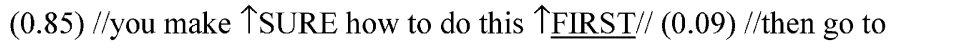 \\
\hline 13 & STOCK $\underline{\mathrm{ROOM}} / /(0.85) / /$ and er GET a $\underline{\mathrm{SAM}} \mathrm{ple} / /(0.56) / /$ because this \\
\hline 14 & TIME limit is a-// (0.5) //you $\uparrow D O N ' T$ get SAMple// (0.2) //after i've \\
\hline 15 & FINished this $\downarrow \underline{\mathrm{TEST}} / / / /(1.0) / / / / \mathrm{SO} / /(0.98) / /$ you get $\uparrow \mathrm{TWO} \uparrow \underline{\mathrm{SAM}} \mathrm{ple}$ \\
\hline 16 & 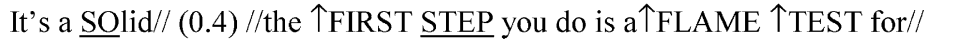 \\
\hline 17 & (0.6) $/ / \uparrow \underline{\text { SOdium ion } / /}$ \\
\hline
\end{tabular}

Fig. 8. Problematic topic structuring and sequence chain boundaries from ITAC- 1's presentation.

chemistry content of the presentation, "the first step you do is a flame test." This recycling of the same introductory statement at the opening of three of the sequence chains followed by unexpected topic shifts contradicts expectations of topic development and does not allow the listener to accurately predict what information may follow the initial focusing marker. In the parallel presentation given by the NS TA, opening statements made in the first three sequence chains clearly divided the organization of the presentation into administrative procedures, ("ok begin about today I'm just gonna go over our unknown analysis scheme"), a topic announcement ("but for our unknown we have seven ions we have to test for"), and the steps in the experimental procedure ("one of the first things we did was a flame test"). 
Sequence chains were also disrupted by what we might think of as typical nonnative speaker linguistic disfluencies resulting from online verbal planning. An example is shown in Fig. 9a from ITAP-1's presentation. The ITA is moving from a review of the overall procedures in the lab, which are quite straightforward, to one particular question near the end of the lab that may confuse the students. The sequence chain opens with a high key comment, "I don't think you will have any problems," which is followed by a series of mid and low key units interspersed with topic length pauses as the ITA redrafts the sequence chain opening. Fig. 9b shows the equivalent section from TAP-1's parallel NS TA presentation. The first sequence chain ends with a mid to low recapitulation statement, "so that's the basic gist of the lab," which is followed by a high to mid key prospective marker, "toward the end though you will be doing something a little bit different."

There was some evidence to suggest that if an organizational framework was in some way pre-imposed on the spoken discourse, this improved nonnative speaker fluency and increased the likelihood of a stronger sequence chain structure. ITAC-1 was the only ITA in the group who prepared the chalkboard prior to his presentation; he listed the major steps the students needed to complete and referred to them during his talk. The opening tone units of three sequence chains and the equivalent board work are shown in Table 5 . The correspondence between the sequence chain openings and the prepared visual material mirror the parallel presentation given by TAC-1 and shown in Table 3. It is possible to speculate that had ITAC-1 also written up some of the administrative details that he intended to cover, he may have avoided some of the problematic structures shown in Fig. 8.

As ITAC-1's presentation illustrates, sequence chain structure across the ITA group was subject to variation both within and between speakers. Within one presentation, an ITA could produce one or more interpretable sequence chains followed

(a)

\begin{tabular}{|ll}
\hline 1 & $/ / / / \mathrm{i} \uparrow \mathrm{DON}$ 'T think $\uparrow$ YOU will have any PROBlem// (0.32) //exCEPT in a// \\
2 & $(1.5) / / \downarrow W E L L$ in A// (2.16) //in a FInal $\downarrow$ AND // (0.98) //er// (0.18) ////one \\
3 & TONE $\uparrow$ CASE is that YOU ARE//...
\end{tabular}

(b)

\begin{tabular}{|ll|}
\hline 1 & $/ /$ so that's the Basic gist of the $\downarrow$ LAB $/ / / /(0.32) / / /$ toward the $\uparrow$ END of the lab \\
2 & THOUGH// (0.23) //UM// (0.6) //you gonna be doing SOMEthing a LITtle bit \\
3 & $\downarrow$ DIFferent// (5.6)
\end{tabular}

Fig. 9. (a) A disrupted sequence chain from ITAP-1's presentation. (b) A parallel sequence chain from TAP-1's presentation. 
Table 5

Visual cues coinciding with sequence chain structure in ITAC-1's presentation

\begin{tabular}{|c|c|}
\hline Opening tone units of three sequence chains (ITAC-2) & Boardwork \\
\hline 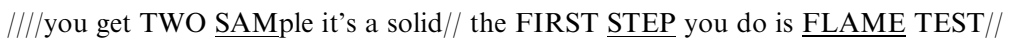 & Flame test orange \\
\hline $\begin{array}{l}\text { ////and the SEcond step I think is VOlatile } \\
\text { Test for nh } 4+/ /\end{array}$ & Volatile test \\
\hline 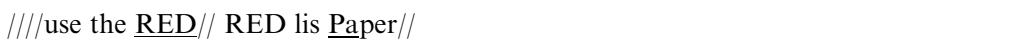 & Red litmus paper \\
\hline
\end{tabular}

by a sequence of structures in which the specific nature of the difficulty varied, but which demonstrated a mismatch of organizational cues on some level. Using both the phonological and transaction based criteria suggested by the analysis of the NS model, only 15 of the 37 sequence chains (41\%) were equivalent in structure and function to those found in the NS data. These included the first sequence chain of each of the six speakers, which often consisted of no more than a brief introduction to the topic.

\subsubsection{Pitch sequence structure}

In the NS TA data, pitch sequence patterning helped to create structural distinctions in the discourse between main and subsidiary content. In the ITA presentations, pitch sequence boundary cues tended not to indicate a principled grouping of tone units. In some cases, prosodic disturbance due to linguistic repairs made an overall pattern difficult to establish, and in others, there appeared to be no apparent motivation for pitch sequence closure by the criteria established in the NS TA model.

In the parallel opening sections from TAC-1 and ITAC-1's chemistry presentations shown in Figs. 4 and 8, the aim of both TAs is to suggest the best method for the students to use to complete an analysis scheme. TAC-1's extract begins with two pitch sequences: an initial high key unit to open the presentation and invite the students up to the board, followed by a mid key expansion. The equivalent extract from ITAC-1's presentation is characterized by high key choices which create sequence chain rather than pitch sequence structures. The units, "this my suggestion for the unknown test, I think it will save you time," together form a similar kind of comment structure to that shown in the NS TA extract. However, the conjunction between the units themselves, and between this comment and the surrounding discourse, is obscured by the consistent return to a high key. The framing and elaboration that are highlighted by the prosodic structure in the NS TA's presentation must work despite this level of linguistic organization in the ITA presentation.

Analysis of the NS TA data showed that effective pitch sequence structuring relied on the principled use of the three key choices available to the speaker, particularly the specific combinations of key and termination choices within tone units. In the ITA data, prosodic structure was largely made up of a series of phrasal units exhibiting either mid-mid or mid-low choices which were collected into sequence chain 


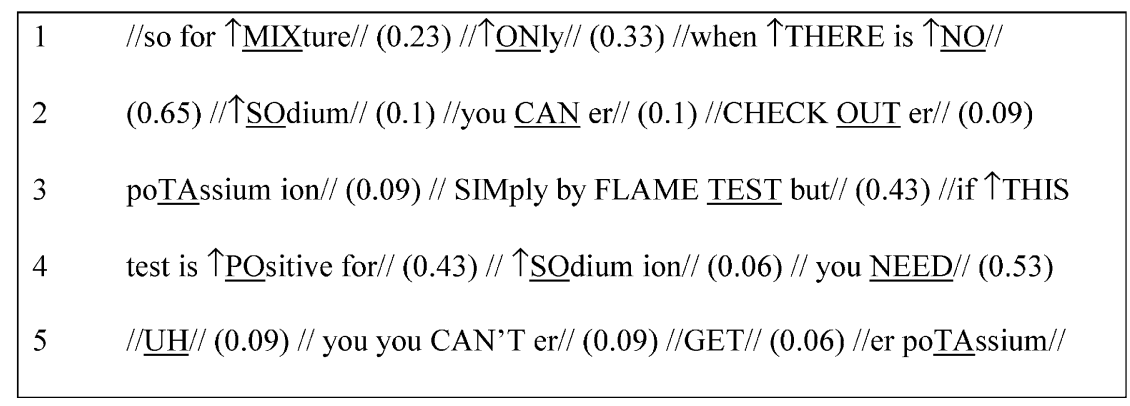

Fig. 10. Minimal tone units and pause structure from ITAC-2's presentation.

structures rather than pitch sequences. Key and termination choices seemed more often determined by other factors such as linguistic coding problems, and, as demonstrated in Fig. 10 from ITAC-2's presentation, these linguistic disfluencies and empty silences created a series of minimal or truncated tone units which constituted the only unit of prosodic organization.

\section{Conclusion}

Using a model of intonation in discourse augmented by a framework designed to map co-occurring semantic and structural cues, this analysis investigated the systematic use of pitch and pause cues to create intonational paragraphs in teaching discourse. Examination of the NS data suggests that speakers intentionally created a hierarchical system of prosodic units to emphasize relationships between semantically related sections of the discourse and highlight information structure. The integrated analysis also supports the proposals previously submitted by Barr (1990) and Tyler et al. (1988) and, most recently, Thompson (2003) for a multiple cuing system operating concurrently at different levels of the discourse structure and used to accentuate subdivisions in the substantive content of the lecture.

Analysis of the ITA data showed that these speakers were unable to consistently manipulate key and tone choices to create intonational paragraphs. There was no simple comparison between the NS TA and ITA data sets; for example, no features that consistently appeared in the NS group were entirely lacking in the ITA group. However, when both problematic sequence chain and transaction boundaries were taken into consideration, fewer than half the sequence chains found in the ITA presentations matched those found in the NS TA data. There is little evidence of a structured prosodic unit between the sequence chain and the tone unit, suggesting that the ITAs may be unable to make the finer distinctions between key choices needed for the pitch sequence structure. Also contributing to the lack of clear pitch sequence structuring was the overall narrower pitch range established for this group by instrumental analysis. Finally, identification of prosodic units was also made more difficult by the presence of a large number of prominent constituents, which made salient key and termination choices difficult to appraise. 


\section{Applications to ITA program instruction}

Teaching discourse in the science laboratory or math discussion class is often described as more interactive and "context-embedded" than that found in lecture presentations (Myers, 1994). TAs frequently adopt a more informal register, and presentations may be characterized by more teacher immediacy behaviors, such as the use of inclusive pronouns and motivational techniques to relieve student anxiety (Rounds, 1987). This does not favor the ITA who is likely to have far less control over register variation than their native speaker counterpart. ITAs may also misunderstand how this more informal pedagogical setting is created. They may view these assignments as requiring less preparation time than they would give to a lecture class. In fact, the NS TA monologues analyzed here are quite complex in their informational composition. As Jacobson (1986: 177) notes in his discussion of physics prelab presentations, TAs tend to move back and forth between different kinds of content fairly quickly, and this presentation may be students' only source of information such as corrections in the lab manual or changes in procedure. Fig. 4 from TAC-1's chemistry presentation illustrated how these different levels of content can be indicated by systematic interaction between lexico-grammatical markers, prosodic composition, and topic structure.

Requiring ITAs to script or rehearse their presentations to the point of memorization is not the answer. This is more likely to hamstring the ITA, who may become less able to respond to real time interaction in the classroom. In addition, the intonation structure that characterizes read speech is qualitatively different from that of prepared or spontaneous speech and may disengage the ITA even further from the unfolding context (Brazil, 1992). Rather, raising conscious awareness of this level of discourse structure and providing opportunities for practice can benefit ITAs in their development of these skills. Currently, little may be done in ITA programs to address areas of linguistic competence such as pitch range or pause structure, as they are often perceived to be less crucial for functional competence than lexical or syntactic marking strategies. Within the integrated framework proposed here, however, prosodic cues contribute independently to the structure of the discourse, and they cannot be circumvented without a reduction in comprehensibility.

A critical feature of ITA discourse which affected a range of prosodic structuring devices was an overall reduction in pitch range. Mennen (1998) suggests that a significantly narrower pitch range in L2 speakers may be related to a lack of confidence in the new language. This can be directly addressed in ITA instruction through the use of standard exercises from theater training designed to increase confidence and encourage speakers to explore their voice range. Vocal warm-ups such as "call and response" exercises require students to produce an extended pitch range as they practice calling a person from a distance (Lochwyn, 1999; Wessels \& Lawrence, 1992).

It may also be the case that while traditional tone or pause units are related to universal cognitive constraints, the organization of longer structural units may be a learned skill and subject to crosslinguistic variation (Chafe, 1994; Wichmann, 2000). Thus, construction of intonational paragraphs could be usefully included in training 
curricula. While scripted or read-aloud speech is not advocated, the analysis preliminarily suggests that effective intonational paragraphing is more likely to appear in teaching discourse that has been to some extent prepared, i.e. the ITA has considered board work and has some sense of overall organization. This preparation may help to reduce the effects of online verbal planning; therefore, we should continue to promote the use of the blackboard and to encourage ITAs to prepare even short prelab presentations in order to maximize comprehensibility for undergraduate native speaker hearers.

Although this study does not directly measure student comprehension of ITA or NS TA discourse, it contributes to a body of research suggesting that organizational structure is a strong predictor of the effectiveness of academic lectures (DudleyEvans, 1994; Tyler et al., 1988; Tyler \& Davies, 1990). Within this field of inquiry, the identification and interpretation of intonational paragraphs is a relatively recent undertaking. The analyses discussed here, however, suggest that it is a potentially powerful organizational tool for L2 speakers who are required to produce complex and lengthy presentations for native speaker consumption.

\section{Acknowledgements}

I thank the participants of SECOL LXIII, Richard Cauldwell, and two anonymous reviewers for their helpful comments on earlier versions of this article. Any errors that remain are entirely my own. This article derives from the author's unpublished doctoral dissertation, An Analysis of the Prosodic Systems in the Classroom Discourse of NS and NNS TAs.

\section{Appendix A}

\section{Transcription conventions}

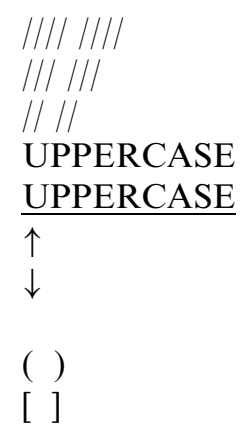

sequence chain boundary

pitch sequence boundary

tone unit boundary

onset syllable

tonic syllable

high key or termination

low key or termination

mid key indicated by the absence of an arrow pause length

topic length pauses coinciding with board work 


\section{Appendix B}

An initial model of the interaction between intonational structure and co-occurring structural boundaries in NS teaching discourse

Intonational structure

////Sequence Chain////

A pitch defined conceptual unit bounded by a high key or lecturing frame and low termination
Co-occurring structural features

\section{Transaction}

A semantically coherent unit with a unifying topic, an introductory topic expression and bounded by prospective and retrospective markers; may coincide with pauses of 0.8 seconds or longer

A semantically coherent group of tone units; Pitch sequence structure distinguishes between main (informative) content and subsidiary (glosses and asides) content

Tone units may coincide with articulartory or short pauses and syntactic boundaries

Onset Key Choice

Tonic Syllable

Termination Choice

Low: Equative

Mid: Additive

High: Contrastive

or Particularized

\section{References}

Anderson-Hsieh, J., Johnson, R., \& Koehler, K. (1992). The relationship between native speaker judgements of nonnative pronunciation and deviance in segmentals, prosody and syllable structure. Language Learning, 42, 529-555.

Anderson-Hsieh, J., \& Venkatagiri, H. (1995). Syllable duration and pausing in the speech of Chinese ESL speakers. TESOL Quarterly, 42, 807-812. 
Axelson, E., \& Madden, C. (1994). Discourse strategies for ITAs across instructional contexts. In C. Madden, \& C. Myers (Eds.), Discourse and performance of international teaching assistants (pp. 153186). Alexandria, VA: TESOL.

Barr, P. (1990). The role of discourse intonation in lecture comprehension. In M. Hewings (Ed.), Papers in discourse intonation (pp. 5-21). Birmingham, UK: University of Birmingham, English Language Research.

Beckman, M. (1997). A typology of spontaneous speech. In Y. Sagisaka, N. Campbell, \& N. Higuchi (Eds.), Computing prosody (pp. 7-26). New York: Springer Verlag.

Bolinger, D. (1988). Intonation and its uses: melody in grammar and discourse. London: Edward Arnold.

Brazil, D. (1986). The communicative value of intonation in English. Birmingham, UK: University of Birmingham, English Language Research.

Brazil, D. (1992). Listening to people reading. In M. Coulthard (Ed.), Advances in spoken discourse analysis (pp. 209-241). London: Routledge.

Brazil, D. (1997). The communicative value of intonation in English. Cambridge: Cambridge University Press.

Brown, G. (1977). Listening to spoken English. London: Longman.

Brown, G., Currie, K., \& Kenworthy, J. (1980). Questions of intonation. London: Croom-Helm.

Brown, G., \& Yule, G. (1983). Discourse analysis. Cambridge: Cambridge University Press.

Chafe, W. (1994). Discourse, consciousness, and time. Chicago: University of Chicago Press.

Coulthard, M., Montgomery, M. (Eds.). (1981). Studies in discourse analysis. London: Thomas Litho Press.

Couper-Kuhlen, E. (1986). An introduction to English prosody. Tubingen: Max Niemeyer-Verlag.

Cruttenden, A. (1997). Intonation. New York: Cambridge University Press.

Crystal, D. (1969). Prosodic systems and intonation in English. London: Cambridge University Press.

Crystal, D. (1987). The Cambridge encyclopedia of language. Cambridge: Cambridge University Press.

Cutler, A., Dahan, D., \& Donselaar, W. (1997). Prosody in the comprehension of spoken language: a literature review. Language and Speech, 40, 141-201.

Dudley-Evans, T. (1994). Variations in the discourse patterns favored by different disciplines and their pedagogical implications. In J. Flowerdew (Ed.), Academic listening: research perspectives (pp. 146158). Cambridge: Cambridge University Press.

Educational Testing Service. (1996). SPEAK rater training guide. Princeton, NJ: ETS.

Griffiths, R. (1991). Pausological research in an L2 context: a rationale, and review of selected studies. Applied Linguistics, 12, 345-364.

Halliday, M. (1967). Intonation and grammar in British English. The Hague: Mouton.

Jacobson, W. (1986). An assessment of the communicative needs of nonnative speakers of English in an undergraduate physics lab. English for Specific Purposes, 5, 173-187.

Lehiste, I. (1979). Perception of sentence and paragraph boundaries. In B. Lindblom, \& S. Ohman (Eds.), Frontiers of speech communication research (pp. 191-201). New York: Academic Press.

Lochwyn, T. (1999). Theater exercises in the ESL classroom. Unpublished paper.

Madden, C., \& Myers, C. (Eds.). (1994). Discourse and performance of international teaching assistants. Arlington, VA: TESOL Inc.

Mennen, I. (1998). Can language learners ever acquire the intonation of a second language? In Proceedings of the ESCA workshop on speech technology in language learning (pp. 17-20). Marholmen, Sweden: International Speech Communication Association.

Myers, C. (1994). Question-based discourse in science labs: issues for ITAs. In C. Madden, \& C. Myers (Eds.), Discourse and performance of international teaching assistants (pp. 83-102). Arlington, VA: TESOL.

Nakajima, S., \& Allen, J. (1993). A study on prosody and discourse structure in cooperative dialogues. Phonetica, 50, 197-210.

Pickering, L. (2001). The role of tone choice in improving ITA communication in the classroom. TESOL Quarterly, 35, 233-255.

Riggenbach, H. (1991). Toward an understanding of fluency: a microanalysis of nonnative speaker conversations. Discourse Processes, 14, 423-441. 
Rounds, P. (1987). Characterizing successful classroom discourse for NNS teaching assistant training. TESOL Quarterly, 21, 643-672.

Schuetze-Coburn, S., Shapley, M., \& Weber, E. (1991). Units of intonation in discourse: a comparison of acoustic and auditory analyses. Language and Speech, 34, 207-234.

Shaw, P. (1994). Discourse competence in a framework for ITA training. In C. Madden, \& C. Myers (Eds.), Discourse and performance of international teaching assistants (pp. 27-51). Alexandria, VA: TESOL.

Sinclair, J., \& Brazil, D. (1982). Teacher talk. London: Oxford University Press.

Swerts, M., \& Geluykens, R. (1993). The prosody of information units in spontaneous monologue. Phonetica, 50, 189-196.

Swerts, M., \& Geluykens, R. (1994). Prosody as a marker of information flow in spoken discourse. Language \& Speech, 37, 21-43.

Tench, P. (1996). The intonation systems of English. London: Cassell.

Thompson, S. E. (2003). Text-structuring in metadiscourse, intonation and the signalling of organization in academic lectures. Journal of English for Academic Purposes, 2, 5-20.

Tyler, A. (1992). Discourse structure and the perception of incoherence in ITA's spoken discourse. TESOL Quarterly, 26, 713-729.

Tyler, A., \& Davies, C. (1990). Cross-linguistic communication missteps. Text, 10, 385-411.

Tyler, A., Jefferies, A., \& Davies, C. (1988). The effect of discourse structuring devices on listener perceptions of coherence in nonnative university teachers' spoken discourse. World Englishes, 7, 101-110.

van Bezooijen, R. (1995). Sociocultural aspects of pitch differences between Japanese and Dutch women. Language \& Speech, 38, 253-265.

Watt, D. (1997). The phonology and semology of intonation in English: an instrumental and systemic perspective. Bloomington: Indiana University Linguistics Club.

Wennerstrom, A. (1994). Intonational meaning in English discourse. Applied Linguistics, 15, 399-421.

Wennerstrom, A. (1997). Discourse intonation and second language acquisition: three genre based studies. Unpublished doctoral dissertation, University of Washington, Seattle.

Wennerstrom, A. (1998). Intonation as cohesion in academic discourse: a study of Chinese speakers of English. Studies in Second Language Acquisition, 20, 1-25.

Wennerstrom, A. (2001). The music of everyday speech. New York: Oxford University Press.

Wessels, C., \& Lawrence, K. (1992). Using drama voice techniques in the teaching of pronunciation. In A. Brown (Ed.), Approaches to pronunciation teaching (pp. 29-37). London: McMillan.

Wichmann, A. (2000). Intonation in text and discourse. Harlow, UK: Longman.

Yule, G. (1980). Speakers' topics and major paratones. Lingua, 52, 33-47.

Lucy Pickerington is Assistant Professor of TESOL and Applied Linguistics at the University of Alabama. Formerly, she taught in the ITA Program at the University of Florida. 